\title{
Electron energy loss spectroscopy of bulk gold with ultrasoft pseudopotentials and the Liouville-Lanczos method
}

\author{
Oleksandr Motornyi ${ }^{1},{ }^{*}$ Nathalie Vast ${ }^{1},{ }^{\dagger}{\text { Iurii } \text { Timrov }^{2}, \text { Oscar Baseggio }}^{3}$, Stefano Baroni ${ }^{3,4}$, and Andrea Dal Corso ${ }^{3,4}$ \\ 1 Laboratoire des Solides Irradiés, CEA/DRF/IRAMIS, École Polytechnique, \\ CNRS, Institut Polytechnique de Paris, 91128 Palaiseau cédex, France \\ ${ }^{2}$ Theory and Simulation of Materials (THEOS), and National Centre for \\ Computational Design and Discovery of Novel Materials (MARVEL), \\ École Polytechnique Fédérale de Lausanne (EPFL), CH-1015 Lausanne, Switzerland \\ 3 Scuola Internazionale Superiore di Studi Avanzati (SISSA), Via Bonomea 265, IT-34136 Trieste, Italy and \\ 4 CNR-IOM DEMOCRITOS, Via Bonomea 265, IT-34136 Trieste, Italy
}

(Dated: November 19, 2021)

\begin{abstract}
The implementation of ultrasoft pseudopotentials into time-dependent density-functional perturbation theory is detailed for both the Sternheimer approach and the Liouville-Lanczos (LL) method, and equations are presented in the scalar relativistic approximation for periodic solids with finite momentum transfer $\mathbf{q}$. The LL method is applied to calculations of the electron energy loss (EEL) spectrum of face-centered cubic bulk Au both at vanishing and finite q. Our study reveals the richness of the physics underlying the various contributions to the density fluctuation in gold. In particular, our calculations suggest the existence in gold of two quasi-separate $5 d$ and $6 s$ electron gasses, each one oscillating with its own frequency at resp. $5.1 \mathrm{eV}$ and $10.2 \mathrm{eV}$. We find that the contribution near $2.2 \mathrm{eV}$ comes from $5 d \rightarrow 6 s$ interband transitions modified by the intraband contribution to the real part of the dielectric function, which we call a mixed excitation.
\end{abstract}

PACS numbers: Condensed-Matter Physics, DFT development, EELS, plasmons, noble metals

\section{INTRODUCTION}

Experiments that probe the dielectric function of finite systems are usefully complemented with ab initio calculations based on time-dependent density-functional theory (TDDFT) [1-3], and sometimes also with manybody perturbation theory, for systems in which excitonic effects are important [4-7] and/or plasmon-phonon interaction is strong, leading to the presence of satellites in the spectrum [8-11].

Various advances have been made in the implementations of TDDFT. On the one hand, fully first-principles nonequilibrium simulations based on real-time timedependent density functional theory (RT-TDDFT) are now accessible [12-14]. On the other hand, the use of perturbation techniques has allowed to progress towards an efficient treatment of the linear response of an electronic system to external perturbations within timedependent (TD) density-functional perturbation theory (DFPT), based on the solution of the Sternheimer equation $[15,16]$. Moreover, the method based on the Lanczos recursion method to solve the quantum Liouville equation, called the Liouville-Lanczos (LL) method, has allowed to speed-up the efficiency of calculations of the TDDFPT spectra, avoiding to solve the Sternheimer equations at each frequency [17-19].

The LL method has been applied to the computation of optical spectra of molecular systems of unprecedented large size [20-23]. Since then, it has been used in the

\footnotetext{
* motorny.sasha@gmail.com

† nathalie.vast@polytechnique.edu
}

framework of many-body perturbation theory to capture the electron-hole interaction in the Bethe-Salpeter equation $[24,25]$. The LL approach to TDDFPT has also been extended to periodic solids and to finite values of the transferred momentum within a norm-conserving pseudopotential (NC-PP) framework to model plasmons [2629]. The aim was to provide a valuable and computationally efficient theoretical tool to complement experiments that probe the dynamical structure factor, as measured in inelastic x-ray scattering (IXS) or the inverse dielectric function, as measured in electron energy loss spectroscopy (EELS) experiments. Moreover, very recently, the LL method has been also generalized to model magnons in magnetic periodic solids [30].

In the present work we discuss the generalization of the LL method for EELS to ultrasoft pseudopotentials (US-PPs) with an application to bulk gold. The objective is to have a tool to investigate plasmons in systems of large size like surfaces with steps (vicinal surfaces)[31]. As the efficiency of the treatment of large surfaces in the slab approach is heavily linked to the size of the plane wave basis set, a significant speed up in the calculations can be obtained when the kinetic energy cut-off of the plane waves is reduced. To this end, US-PPs have been developed [32] about three decades ago to deal with electronic states localized near the nucleus of an atom. Actually the lift of the norm conservation constraint for the pseudo-wavefunctions, and the use of several reference energies for each angular momentum, with the multiprojector scheme inherent to the US-PP formalism, lead to accurate PPs even with a small kinetic energy cut-off for the plane waves. Numerous developments have extended the use of US-PPs in DFPT [15], for instance for 
lattice dynamics [33, 34], electric field perturbations [35], and TDDFPT for optical absorption of molecules [36]. Most of the work has been done for the scalar relativistic (SR) US-PP scheme, but both DFT [37] and DFPT for lattice dynamics [38] have been generalized to the fully relativistic US-PPs scheme, including spin-orbit interaction in the solution of the Sternheimer linear system.

The LL approach is similar to the Sternheimer method, in the sense that it does not need to perform expensive summations over empty states. However, in contrast to the Sternheimer method, there is no need to perform computations at each value of the excitation frequency: this is possible thanks to the use of the recursive Lanczos algorithm, which allows to obtain the charge-density susceptibility on an arbitrarily wide energy range with just one Lanczos chain. Due to the efficiency of the LL approach, it is used in this work and extended to the use of US-PPs. The LL method is then applied to the calculation of the electron energy loss (EEL) spectrum of fcc-Au. Revisiting the EEL spectrum for $\mathbf{q} \rightarrow 0$, we provide a complete characterization and interpretation of all peaks and moreover we provide some more insights into the understanding of the origin of certain excitations.

This paper is organized as follows. We first present the TDDFPT formalism with SR US-PPs as implemented in two ways, the Sternheimer equations and the LL method, for periodic solids and finite momentum transfer (Section II). The LL method with US-PPs is benchmarked against the NC-PPs implementation and the FP-LAPW method for bulk $\mathrm{Au}$, and then we present our results, comparison with the experiments, and discuss the origin of the peaks in the EEL spectra of bulk Au in Section III. Conclusions are drawn in section IV.

\section{TDDFPT FORMALISM WITH ULTRASOFT PSEUDOPOTENTIALS}

In the following, the formalism is detailed for insulators for the sake of simplicity and clarity and the discussion is limited to scalar relativistic PPs. The Reader is referred to ref. 34 for the metallic case in US-PP DFPT. Hartree atomic units are used throughout the paper. For operators we will use a hat on top "^" of the symbol.

\section{A. TDDFT equations in the US-PP scheme}

Both EELS and IXS cross-sections are proportional to $S(\mathbf{q}, \omega)$, the dynamical structure factor per unit volume of the solid, where $\mathbf{q}$ is the transferred momentum and $\omega$ is the energy loss. $S(\mathbf{q}, \omega)$ is proportional to the imaginary part of the charge-density susceptibility $\chi(\mathbf{q}, \mathbf{q}, \omega)$ :

$$
S(\mathbf{q}, \omega)=-\frac{1}{\pi} \operatorname{Im} \chi(\mathbf{q}, \mathbf{q}, \omega) .
$$

The charge-density susceptibility $\chi\left(\mathbf{r}, \mathbf{r}^{\prime}, \omega\right)$ of a system relates to the charge density induced by an external per- turbing potential [39]:

$$
n^{\prime}(\mathbf{r}, \omega)=\int d^{3} r^{\prime} \chi\left(\mathbf{r}, \mathbf{r}^{\prime}, \omega\right) V_{\mathrm{ext}}^{\prime}\left(\mathbf{r}^{\prime}, \omega\right)
$$

Therefore, when the external perturbation is an electron (plane wave) with a fixed momentum $\mathbf{q}$ and the external perturbing potential is $V_{\text {ext }}^{\prime}\left(\mathbf{r}^{\prime}, \omega\right)=e^{i \mathbf{q} \cdot \mathbf{r}^{\prime}}$ we see from Eq. (2) that the charge-density response at frequency $\omega$ reads:

$$
n^{\prime}(\mathbf{r}, \omega)=\chi(\mathbf{r}, \mathbf{q}, \omega)
$$

and the subsequent Fourier transform of $n^{\prime}(\mathbf{r}, \omega)$ at $\mathbf{q}$ is the requested charge-density susceptibility $\chi(\mathbf{q}, \mathbf{q}, \omega)$.

In the time domain, the electronic charge density in Vanderbilt's US-PP scheme [32] reads:

$$
n(\mathbf{r}, t)=2 \sum_{\mathbf{k}, i}\left\langle\psi_{\mathbf{k}, i}(t)|\hat{K}(\mathbf{r})| \psi_{\mathbf{k}, i}(t)\right\rangle,
$$

where the index $\mathbf{k}$ runs over the points in the Brillouin zone (BZ), the index $i$ runs over the occupied Kohn-Sham (KS) wavefunctions, and the factor 2 accounts for spin degeneracy. In Eq. (4), $\hat{K}(\mathbf{r})$ is a nonlocal operator at every point in space $\mathbf{r}$ and in coordinate representation it would be $K\left(\mathbf{r}, \mathbf{r}_{1}, \mathbf{r}_{2}\right)$ [34]. In the NC-PP case, $K\left(\mathbf{r}, \mathbf{r}_{1}, \mathbf{r}_{2}\right)$ is simply $\delta\left(\mathbf{r}-\mathbf{r}_{1}\right) \delta\left(\mathbf{r}-\mathbf{r}_{2}\right)$, with $\delta$ the Dirac distribution, and hence Eq. (4) reduces to $n(\mathbf{r}, t)=2 \sum_{\mathbf{k}, i}\left|\psi_{\mathbf{k}, i}(\mathbf{r}, t)\right|^{2}$. Instead, in the US-PP case, it contains the so-called augmentation term due to the lift of the norm conservation constraint on the pseudo wavefunction:

$$
\hat{K}(\mathbf{r})=|\mathbf{r}\rangle\left\langle\mathbf{r}\left|+\sum_{I m n} Q_{m n}^{\gamma(I)}\left(\mathbf{r}-\mathbf{R}_{I}\right)\right| \beta_{m}^{I}\right\rangle\left\langle\beta_{n}^{I}\right|,
$$

where the index $I$ runs over atoms, $\gamma(I)$ is the type of atom $I, Q_{m n}^{\gamma(I)}\left(\mathbf{r}-\mathbf{R}_{I}\right)$ and $\left\langle\mathbf{r} \mid \beta_{m}^{I}\right\rangle \equiv \beta_{m}^{\gamma(I)}\left(\mathbf{r}-\mathbf{R}_{I}\right)$ are the augmentation functions and projector functions of atom $I$ centered at $\mathbf{R}_{I}$, respectively, and the indices $m$ and $n$ run over all the projectors of the atom I. $Q_{m n}^{\gamma(I)}(\mathbf{r})$ are calculated by pseudizing the difference $\varphi_{m}^{\gamma(I) *}(\mathbf{r}) \varphi_{n}^{\gamma(I)}(\mathbf{r})-\phi_{m}^{\gamma(I) *}(\mathbf{r}) \phi_{n}^{\gamma(I)}(\mathbf{r})$ so that they are easily expanded in plane waves but conserve the multipole moments. Here $\varphi_{m}^{\gamma(I)}(\mathbf{r})$ and $\phi_{n}^{\gamma(I)}(\mathbf{r})$ are the all-electron and pseudo partial waves, respectively [32]. The augmentation functions and the projector functions are localized in spheres about each atom $I$ and are generated together with the US-PP.

In the US-PP scheme of TDDFPT, the TD KS equations read: [40]

$$
i \hat{S} \frac{\partial\left|\psi_{\mathbf{k}, i}(t)\right\rangle}{\partial t}=\hat{H}_{\mathrm{KS}}(t)\left|\psi_{\mathbf{k}, i}(t)\right\rangle
$$

where $\hat{S}$ is an overlap operator:

$$
\hat{S}=1+\sum_{I m n} q_{m n}^{\gamma(I)}\left|\beta_{m}^{I}\right\rangle\left\langle\beta_{n}^{I}\right|
$$


whose coefficients are defined as $q_{m n}^{\gamma(I)}=\int d^{3} r Q_{m n}^{\gamma(I)}(\mathbf{r})$. In Eq. (6), $\hat{H}_{\mathrm{KS}}(t)$ is the TD KS Hamiltonian which reads:

$$
\hat{H}_{\mathrm{KS}}(t)=\hat{H}^{\circ}+\hat{V}^{\prime}(t)
$$

It is a nonlocal operator, where $\hat{H}^{\circ}$ is the Hamiltonian of the unperturbed system, and $\hat{V}^{\prime}(t)$ is the TD linearized potential:

$$
\hat{V}^{\prime}(t)=\int d^{3} r^{\prime}\left[V_{\mathrm{ext}}^{\prime}\left(\mathbf{r}^{\prime}, t\right)+V_{\mathrm{Hxc}}^{\prime}\left(\mathbf{r}^{\prime}, t\right)\right] \hat{K}\left(\mathbf{r}^{\prime}\right)
$$

Here, $V_{\text {ext }}^{\prime}(\mathbf{r}, t)$ is the external TD perturbing potential, and $V_{\mathrm{Hxc}}^{\prime}(\mathbf{r}, t)$ is the linear-response TD Hartree and exchange-and-correlation ( $\mathrm{Hxc}$ ) potential. At variance with the NC-PPs case, in the US-PPs case the operator $\hat{V}^{\prime}(t)$ is nonlocal, because $\hat{K}(\mathbf{r})$ is nonlocal [see Eq. (5)]: $\hat{V}^{\prime}(t)$ in the coordinate representation is $V^{\prime}\left(\mathbf{r}_{1}, \mathbf{r}_{2}, t\right)$. We consider a real external perturbation of the form[26]:

$$
\begin{aligned}
V_{\text {ext }}^{\prime}(\mathbf{r}, t) & =\int_{-\infty}^{\infty} d \omega V_{\text {ext }}^{\prime}(\mathbf{r}, \omega) e^{-i \omega t} \\
& =\int_{0}^{\infty} d \omega\left[V_{\text {ext }}^{\prime}(\mathbf{r}, \omega) e^{-i \omega t}+c . c .\right],
\end{aligned}
$$

and compute the linearly induced charge density $n^{\prime}(\mathbf{r}, t)$. The TD KS wavefunctions can be developed to first order as:

$$
\left|\psi_{\mathbf{k}, i}(t)\right\rangle=e^{-i \varepsilon_{\mathbf{k}, i} t}\left[\left|\psi_{\mathbf{k}, i}^{\circ}\right\rangle+\left|\psi_{\mathbf{k}, i}^{\prime}(t)\right\rangle\right],
$$

so that the TD charge density becomes $n(\mathbf{r}, t)=n^{\circ}(\mathbf{r})+$ $n^{\prime}(\mathbf{r}, t)$, where $n^{\circ}(\mathbf{r})$ is the unperturbed charge density. Going from time to frequency domain by Fourier transforming all quantities, the Fourier transform of $n^{\prime}(\mathbf{r}, t)$ reads:

$$
\begin{aligned}
n^{\prime}(\mathbf{r}, \omega) & =2 \sum_{\mathbf{k}, i}\left\{\left\langle\psi_{\mathbf{k}, i}^{\circ} \mid \mathbf{r}\right\rangle\left\langle\mathbf{r} \mid \psi_{\mathbf{k}, i}^{\prime}(\omega)\right\rangle+\left\langle\psi_{\mathbf{k}, i}^{\prime}(-\omega) \mid \mathbf{r}\right\rangle\left\langle\mathbf{r} \mid \psi_{\mathbf{k}, i}^{\circ}\right\rangle\right. \\
& \left.+\sum_{I m n} Q_{m n}^{\gamma(I)}\left(\mathbf{r}-\mathbf{R}_{I}\right)\left[\left\langle\psi_{\mathbf{k}, i}^{\circ} \mid \beta_{m}^{I}\right\rangle\left\langle\beta_{n}^{I} \mid \psi_{\mathbf{k}, i}^{\prime}(\omega)\right\rangle+\left\langle\psi_{\mathbf{k}, i}^{\prime}(-\omega) \mid \beta_{m}^{I}\right\rangle\left\langle\beta_{n}^{I} \mid \psi_{\mathbf{k}, i}^{\circ}\right\rangle\right]\right\},
\end{aligned}
$$

where $\left|\psi_{\mathbf{k}, i}^{\prime}(\omega)\right\rangle$ is the Fourier transform of $\left|\psi_{\mathbf{k}, i}^{\prime}(t)\right\rangle$. The formalism of the NC-PPs can be recovered by setting the augmentation terms to zero, i.e. only the first row in the equation above will remain [26].

In a periodic solid it is convenient to use the Bloch theorem by writing the KS wavefunctions as: $\left\langle\mathbf{r} \mid \psi_{\mathbf{k}, i}^{\circ}\right\rangle=$ $e^{i \mathbf{k} \cdot \mathbf{r}}\left\langle\mathbf{r} \mid u_{\mathbf{k}, i}^{\circ}\right\rangle$, where $\left\langle\mathbf{r} \mid u_{\mathbf{k}, i}^{\circ}\right\rangle$ is a lattice-periodic function. The total external perturbing potential $V_{\text {ext }}^{\prime}(\mathbf{r}, \omega)$ can be written as a sum of the Fourier monochromatic q components, i.e as:

$$
V_{\mathrm{ext}}^{\prime}(\mathbf{r}, \omega)=\sum_{\mathbf{q}} e^{i \mathbf{q} \cdot \mathbf{r}} v_{\mathrm{ext}, \mathbf{q}}^{\prime}(\mathbf{r}, \omega)
$$

where $v_{\text {ext,q }}^{\prime}(\mathbf{r}, \omega)$ is the lattice-periodic part of the perturbation. In EELS, for a beam of incoming electrons, each of which undergoing a certain momentum transfer $\mathbf{q}$, the perturbation is $v_{\text {ext,q }}^{\prime}(\mathbf{r}, \omega)=1$ for a given $\mathbf{q}$, and zero for all the others. In this case, the response $\mathrm{KS}$ wavefunctions can be written as:

$$
\left\langle\mathbf{r} \mid \psi_{\mathbf{k}, i}^{\prime}(\omega)\right\rangle=\sum_{\mathbf{q}} e^{i(\mathbf{k}+\mathbf{q}) \cdot \mathbf{r}}\left\langle\mathbf{r} \mid u_{\mathbf{k}+\mathbf{q}, i}^{\prime}(\omega)\right\rangle .
$$

The response charge density and response HXC potential can be decomposed in the same way:

$$
n^{\prime}(\mathbf{r}, \omega)=\sum_{\mathbf{q}} e^{i \mathbf{q} \cdot \mathbf{r}} n_{\mathbf{q}}^{\prime}(\mathbf{r}, \omega),
$$

where $n_{\mathbf{q}}^{\prime}(\mathbf{r}, \omega)$ is the lattice-periodic part. After introducing the identity $1=\hat{P}_{v}+\hat{P}_{c}$ in Eq. (12), with $\hat{P}_{c}$ (resp. $\hat{P}_{v}$ ) the projectors onto the conduction (resp. valence) states, it can be shown that $n_{\mathbf{q}}^{\prime}(\mathbf{r}, \omega)$ reads:

$$
\begin{aligned}
n_{\mathbf{q}}^{\prime}(\mathbf{r}, \omega) & =2 \sum_{\mathbf{k}, i}\left\{\left\langle u_{\mathbf{k}, i}^{\circ} \mid \mathbf{r}\right\rangle\left\langle\mathbf{r} \mid \hat{P}_{c}^{\mathbf{k}+\mathbf{q}} u_{\mathbf{k}+\mathbf{q}, i}^{\prime}(\omega)\right\rangle+\left\langle u_{\mathbf{k}, i}^{\circ} \mid \mathbf{r}\right\rangle\left\langle\mathbf{r} \mid \hat{P}_{c}^{\mathbf{k}+\mathbf{q}} u_{-\mathbf{k}-\mathbf{q}, i}^{\prime *}(-\omega)\right\rangle\right. \\
& \left.+\sum_{s m n} \tilde{Q}_{m n}^{\gamma(s), \mathbf{q}}\left(\mathbf{r}-\boldsymbol{\tau}_{s}\right)\left\langle\psi_{\mathbf{k}, i} \mid \beta_{m}^{s}\right\rangle\left[A_{n}^{s, \mathbf{k}+\mathbf{q}, i}(\omega)+B_{n}^{s,-\mathbf{k}-\mathbf{q}, i}(-\omega)\right]\right\}
\end{aligned}
$$

where

$$
A_{n}^{s, \mathbf{k}+\mathbf{q}, i}(\omega)=\int d^{3} r \beta_{n}^{s *}\left(\mathbf{r}-\boldsymbol{\tau}_{s}\right) e^{i(\mathbf{k}+\mathbf{q}) \cdot \mathbf{r}}\left\langle\mathbf{r} \mid \hat{P}_{c}^{\mathbf{k}+\mathbf{q}} u_{\mathbf{k}+\mathbf{q}, i}^{\prime}(\omega)\right\rangle
$$




$$
B_{n}^{s,-\mathbf{k}-\mathbf{q}, i}(-\omega)=\int d^{3} r \beta_{n}^{s *}\left(\mathbf{r}-\boldsymbol{\tau}_{s}\right) e^{i(\mathbf{k}+\mathbf{q}) \cdot \mathbf{r}}\left\langle\mathbf{r} \mid \hat{P}_{c}^{\mathbf{k}+\mathbf{q}} u_{-\mathbf{k}-\mathbf{q}, i}^{\prime *}(-\omega)\right\rangle,
$$

and

$$
\tilde{Q}_{m n}^{\gamma(s), \mathbf{q}}\left(\mathbf{r}-\boldsymbol{\tau}_{s}\right)=e^{-i \mathbf{q} \cdot \mathbf{r}} \sum_{l} e^{i \mathbf{q} \cdot \mathbf{R}_{l}} Q_{m n}^{\gamma(s)}\left(\mathbf{r}-\mathbf{R}_{l}-\boldsymbol{\tau}_{s}\right)
$$

In Eqs. (16) - (19), and in the following, we use the fact that atomic positions in a periodic solid can be indicated as $\mathbf{R}_{I}=\mathbf{R}_{l}+\boldsymbol{\tau}_{s}$, where $\mathbf{R}_{l}$ is a Bravais lattice vector and $\boldsymbol{\tau}_{s}$ is the position of the atom in one unit cell $(I=\{l, s\})$. The projector onto the conduction manifold $\hat{P}_{c}^{\mathbf{k}+\mathbf{q}}$ is defined as $[15,34]$ :

$$
\hat{P}_{c}^{\mathbf{k}+\mathbf{q}}=1-\sum_{j}^{\mathrm{occ}}\left|u_{\mathbf{k}+\mathbf{q}, j}^{\circ}\right\rangle\left\langle u_{\mathbf{k}+\mathbf{q}, j}^{\circ}\right| \hat{S}_{\mathbf{k}+\mathbf{q}}
$$

where the sum over $j$ runs over the occupied states. The overlap operator $\hat{S}_{\mathbf{k}+\mathbf{q}}$ in the coordinate representation is defined as:

$$
\left\langle\mathbf{r}\left|\hat{S}_{\mathbf{k}+\mathbf{q}}\right| \mathbf{r}^{\prime}\right\rangle=e^{-i(\mathbf{k}+\mathbf{q}) \cdot \mathbf{r}}\left\langle\mathbf{r}|\hat{S}| \mathbf{r}^{\prime}\right\rangle e^{i(\mathbf{k}+\mathbf{q}) \cdot \mathbf{r}^{\prime}}
$$

We have used the same notations as in Eq. (34) of ref. 15.

\section{B. The Sternheimer equations in the US-PP scheme}

The responses of the wavefunctions, $\left|\hat{P}_{c}^{\mathbf{k}+\mathbf{q}} u_{\mathbf{k}+\mathbf{q}, i}^{\prime}(\omega)\right\rangle$ and $\left|\hat{P}_{c}^{\mathbf{k}+\mathbf{q}} u_{-\mathbf{k}-\mathbf{q}, i}^{\prime *}(-\omega)\right\rangle$, that appears in Eq. (16) can be obtained, within TDDFPT, by solving the Sternheimer equations. In refs. 26-29 these equations have been derived with NC-PPs. By inserting Eq. (11) in Eq. (6), using the Bloch theorem, and making a Fourier transformation from the time domain to the frequency domain, we obtain the first Sternheimer equation for the latticeperiodic part of the response KS wavefunctions in the
US-PP scheme:

$$
\begin{aligned}
{\left[\hat{H}_{\mathbf{k}+\mathbf{q}}^{\circ}-\left(\varepsilon_{\mathbf{k}, i}+\omega\right) \hat{S}_{\mathbf{k}+\mathbf{q}}\right] \mid } & \left|\hat{P}_{c}^{\mathbf{k}+\mathbf{q}} u_{\mathbf{k}+\mathbf{q}, i}^{\prime}(\omega)\right\rangle= \\
& -\hat{P}_{c}^{\dagger \mathbf{k}+\mathbf{q}} \hat{v}_{\mathbf{q}}^{\prime}(\omega)\left|u_{\mathbf{k}, i}^{\circ}\right\rangle .
\end{aligned}
$$

The Hermitian conjugation in the operator $\hat{P}_{c}^{\dagger \mathbf{k}+\mathbf{q}}$ comes from the presence of the overlap matrix with US-PPs and has no equivalence in the NC-PP case. The operator $\hat{H}_{\mathbf{k}+\mathbf{q}}^{\circ}$ is defined as [15]:

$$
\left\langle\mathbf{r}\left|\hat{H}_{\mathbf{k}+\mathbf{q}}^{\circ}\right| \mathbf{r}^{\prime}\right\rangle=e^{-i(\mathbf{k}+\mathbf{q}) \cdot \mathbf{r}}\left\langle\mathbf{r}\left|\hat{H}^{\circ}\right| \mathbf{r}^{\prime}\right\rangle e^{i(\mathbf{k}+\mathbf{q}) \cdot \mathbf{r}^{\prime}} .
$$

Considering the complex conjugate of Eq. (22) at $-\mathbf{k}$ for a perturbation with $-\mathbf{q}$ and $-\omega$, and by using the timereversal symmetry, we obtain the second Sternheimer equation:

$$
\begin{aligned}
{\left[\hat{H}_{\mathbf{k}+\mathbf{q}}^{\circ}-\left(\varepsilon_{\mathbf{k}, i}-\omega\right) \hat{S}_{\mathbf{k}+\mathbf{q}}\right] \mid } & \left.\hat{P}_{c}^{\mathbf{k}+\mathbf{q}} u_{-\mathbf{k}-\mathbf{q}}^{\prime *}(-\omega)\right\rangle= \\
& -\hat{P}_{c}^{\dagger \mathbf{k}+\mathbf{q}} \hat{v}_{\mathbf{q}}^{\prime}(\omega)\left|u_{\mathbf{k}, i}^{\circ}\right\rangle .
\end{aligned}
$$

Here, due to time-reversal symmetry, $v_{\mathrm{Hxc},-\mathbf{q}}^{*}(\mathbf{r},-\omega)=$ $v_{\mathrm{Hxc}, \mathbf{q}}^{\prime}(\mathbf{r}, \omega)$, and we also used the fact that $\hat{K}(\mathbf{r})$ is a real operator. Except for the presence of the overlap matrix $\hat{S}_{\mathbf{k}+\mathbf{q}}$, Eqs. (22) and (24) are formally similar to the NC-PP case presented in refs. 26-29. Note, however, that in the US-PP case, the frequency-dependent potential on the right-hand side of Eqs. (22) and (24) is a nonlocal operator and has a more complex form due to the augmentation terms. Consequently, the object $\hat{v}_{\mathbf{q}}^{\prime}(\omega)\left|u_{\mathbf{k}, i}^{\circ}\right\rangle$ must be interpreted as a shorthand notation for the lattice-periodic part of $\hat{V}^{\prime}(\omega)\left|\psi_{\mathbf{k}, i}^{\circ}\right\rangle$ :

$$
\begin{aligned}
& \left\langle\mathbf{r}\left|\hat{v}_{\mathbf{q}}^{\prime}(\omega)\right| u_{\mathbf{k}, i}^{\circ}\right\rangle \equiv e^{-i(\mathbf{k}+\mathbf{q}) \cdot \mathbf{r}} \\
& \times\left\langle\mathbf{r}\left|\int d^{3} r^{\prime} e^{i \mathbf{q} \cdot \mathbf{r}^{\prime}}\left(1+\hat{v}_{\mathrm{Hxc}, \mathbf{q}}^{\prime}\left(\mathbf{r}^{\prime}, \omega\right)\right) \hat{K}\left(\mathbf{r}^{\prime}\right)\right| \psi_{\mathbf{k}, i}^{\circ}\right\rangle .
\end{aligned}
$$

By inserting the expression of $\hat{K}\left(\mathbf{r}^{\prime}\right)$ [see Eq. (5)] in Eq. (25), we can rewrite Eqs. (22) and (24) as:

$$
\begin{aligned}
{\left[\hat{H}_{\mathbf{k}+\mathbf{q}}^{\circ}-\left(\varepsilon_{\mathbf{k}, i}+\omega\right) \hat{S}_{\mathbf{k}+\mathbf{q}}\right]\left|\hat{P}_{c}^{\mathbf{k}+\mathbf{q}} u_{\mathbf{k}+\mathbf{q}, i}^{\prime}(\omega)\right\rangle=} & -\hat{P}_{c}^{\dagger \mathbf{k}+\mathbf{q}}\left[\left|u_{\mathbf{k}, i}^{\circ}\right\rangle+\hat{v}_{\mathrm{Hxc}, \mathbf{q}}^{\prime}(\omega)\left|u_{\mathbf{k}, i}^{\circ}\right\rangle\right. \\
& \left.+\sum_{s m n}\left({ }^{0} I_{m n}^{s, \mathbf{q}}+{ }^{3} I_{m n}^{s, \mathbf{q}}(\omega)\right)\left|\beta_{m}^{s, \mathbf{k}+\mathbf{q}}\right\rangle\left\langle\beta_{n}^{s} \mid \psi_{\mathbf{k}, i}^{\circ}\right\rangle\right], \\
{\left[\hat{H}_{\mathbf{k}+\mathbf{q}}^{\circ}-\left(\varepsilon_{\mathbf{k}, i}-\omega\right) \hat{S}_{\mathbf{k}+\mathbf{q}}\right]\left|\hat{P}_{c}^{\mathbf{k}+\mathbf{q}} u_{-\mathbf{k}-\mathbf{q}, i}^{\prime *}(-\omega)\right\rangle=} & -\hat{P}_{c}^{\dagger \mathbf{k}+\mathbf{q}}\left[\left|u_{\mathbf{k}, i}^{\circ}\right\rangle+\hat{v}_{\mathrm{Hxc}, \mathbf{q}}^{\prime}(\omega)\left|u_{\mathbf{k}, i}^{\circ}\right\rangle\right. \\
& \left.+\sum_{s m n}\left({ }^{0} I_{m n}^{s, \mathbf{q}}+{ }^{3} I_{m n}^{s, \mathbf{q}}(\omega)\right)\left|\beta_{m}^{s, \mathbf{k}+\mathbf{q}}\right\rangle\left\langle\beta_{n}^{s} \mid \psi_{\mathbf{k}, i}^{\circ}\right\rangle\right],
\end{aligned}
$$

where we defined ${ }^{0} I_{m n}^{s, \mathbf{q}}$ as:

$$
{ }^{0} I_{m n}^{s, \mathbf{q}}=\int d^{3} r e^{i \mathbf{q} \cdot \mathbf{r}} Q_{m n}^{\gamma(s)}\left(\mathbf{r}-\boldsymbol{\tau}_{s}\right)
$$


and ${ }^{3} I_{m n}^{s, \mathbf{q}}(\omega)$ as:

$$
{ }^{3} I_{m n}^{s, \mathbf{q}}(\omega)=\int d^{3} r e^{i \mathbf{q} \cdot \mathbf{r}} v_{\mathrm{Hxc}, \mathbf{q}}^{\prime}(\mathbf{r}, \omega) Q_{m n}^{\gamma(s)}\left(\mathbf{r}-\boldsymbol{\tau}_{s}\right)
$$

We have used the notations ${ }^{0} I_{m n}^{s, \mathbf{q}}$ and ${ }^{3} I_{m n}^{s, \mathbf{q}}(\omega)$ to be consistent with notations in ref. 34. Moreover, we have defined [cf. with Eq. (19)]:

$$
\left\langle\mathbf{r} \mid \beta_{m}^{s, \mathbf{k}+\mathbf{q}}\right\rangle=e^{-i(\mathbf{k}+\mathbf{q}) \cdot \mathbf{r}} \sum_{l} e^{i(\mathbf{k}+\mathbf{q}) \cdot \mathbf{R}_{l}} \beta_{m}^{\gamma(s)}\left(\mathbf{r}-\mathbf{R}_{l}-\boldsymbol{\tau}_{s}\right) .
$$

Note that $\left\langle\beta_{n}^{s} \mid \psi_{\mathbf{k}, i}^{o}\right\rangle=\frac{1}{N}\left\langle\beta_{n}^{s, \mathbf{k}} \mid u_{\mathbf{k}, i}^{o}\right\rangle$, where $N$ is the number of cells of the system, so that Eqs. (26) and (27) are written in terms of lattice-periodic functions. The linear system of Eqs. (26) and (27) can be solved selfconsistently at each frequency $\omega$. The Fourier transform of the lattice-periodic part of the self-consistent response charge-density calculated at $\mathbf{G}=\mathbf{0}$ yields the required susceptibility,

$$
n^{\prime}(\mathbf{q}+\mathbf{G}, \omega)=\sum_{\mathbf{G}^{\prime}} \chi\left(\mathbf{q}+\mathbf{G}, \mathbf{q}+\mathbf{G}^{\prime}, \omega\right) V_{\mathrm{ext}}^{\prime}\left(\mathbf{q}+\mathbf{G}^{\prime}, \omega\right)
$$

and in order to obtain the macroscopic dielectric function we only need terms for which $\mathbf{G}=\mathbf{G}^{\prime}=0$. Finally, the electronic susceptibility is given by Eq. (3).

\section{The quantum Liouville equation in the US-PP scheme}

An alternative approach to the self-consistent solution of the Sternheimer equations at each frequency has been developed using the quantum Liouville equation, the so-called LL method [17, 18, 21, 26-29, 36] that allows a significant reduction of the required computational resources. In this approach one can work in the standard batch representation (SBR), where the response KS wavefunctions are rotated and represented as batches $q_{\mathbf{q}}=\left\{\left|q_{\mathbf{k}+\mathbf{q}, i}\right\rangle\right\}$ and $p_{\mathbf{q}}=\left\{\left|p_{\mathbf{k}+\mathbf{q}, i}\right\rangle\right\}$, where

$$
\begin{aligned}
& \left|q_{\mathbf{k}+\mathbf{q}, i}\right\rangle=\frac{1}{2}\left[\left|\hat{P}_{c}^{\mathbf{k}+\mathbf{q}} u_{\mathbf{k}+\mathbf{q}, i}^{\prime}(\omega)\right\rangle+\left|\hat{P}_{c}^{\mathbf{k}+\mathbf{q}} u_{-\mathbf{k}-\mathbf{q}, i}^{\prime *}(-\omega)\right\rangle\right] \\
& \left|p_{\mathbf{k}+\mathbf{q}, i}\right\rangle=\frac{1}{2}\left[\left|\hat{P}_{c}^{\mathbf{k}+\mathbf{q}} u_{\mathbf{k}+\mathbf{q}, i}^{\prime}(\omega)\right\rangle-\left|\hat{P}_{c}^{\mathbf{k}+\mathbf{q}} u_{-\mathbf{k}-\mathbf{q}, i}^{\prime *}(-\omega)\right\rangle\right] .
\end{aligned}
$$

In the SBR the linearized quantum Liouville equation in the US-PP scheme can be derived by writing the Sternheimer equations (26) and (27) in terms of $\left|q_{\mathbf{k}+\mathbf{q}, i}\right\rangle$ and $\left|p_{\mathbf{k}+\mathbf{q}, i}\right\rangle$. Multiplying both equations by $\hat{S}_{\mathbf{k}+\mathbf{q}}^{-1}$ we obtain (see appendix A):

$$
\left(\begin{array}{cc}
\omega & -\hat{\mathcal{D}}_{\mathbf{q}} \\
-\hat{\mathcal{D}}_{\mathbf{q}}-\hat{\mathcal{K}}_{\mathbf{q}} & \omega
\end{array}\right)\left(\begin{array}{c}
q_{\mathbf{q}} \\
p_{\mathbf{q}}
\end{array}\right)=\left(\begin{array}{c}
0 \\
y_{\mathbf{q}}
\end{array}\right)
$$

where the actions of the operators $\hat{\mathcal{D}}_{\mathbf{q}}$ and $\hat{\mathcal{K}}_{\mathbf{q}}$ on the batches are defined as:

$$
\hat{\mathcal{D}}_{\mathbf{q}} \cdot q_{\mathbf{q}}=\left\{\left(\hat{S}_{\mathbf{k}+\mathbf{q}}^{-1} \hat{H}_{\mathbf{k}+\mathbf{q}}^{\circ}-\varepsilon_{\mathbf{k}, i}\right)\left|q_{\mathbf{k}+\mathbf{q}, i}\right\rangle\right\}
$$

and

$$
\begin{aligned}
\hat{\mathcal{K}}_{\mathbf{q}} \cdot q_{\mathbf{q}}= & \left\{\hat { S } _ { \mathbf { k } + \mathbf { q } } ^ { - 1 } \hat { P } _ { c } ^ { \dagger \mathbf { k } + \mathbf { q } } \left[\hat{v}_{\mathrm{Hxc}, \mathbf{q}}^{\prime}(\omega)\left|u_{\mathbf{k}, i}^{\circ}\right\rangle\right.\right. \\
& \left.\left.+\sum_{s m n}{ }^{3} I_{m n}^{s, \mathbf{q}}(\omega)\left|\beta_{m}^{s, \mathbf{k}+\mathbf{q}}\right\rangle\left\langle\beta_{m}^{s} \mid \psi_{\mathbf{k}, i}^{\circ}\right\rangle\right]\right\} .
\end{aligned}
$$

On the right-hand side of Eq. (34), $y_{\mathbf{q}}$ is the perturbation term which reads:

$$
\begin{aligned}
y_{\mathbf{q}}= & \left\{\hat { S } _ { \mathbf { k } + \mathbf { q } } ^ { - 1 } \hat { P } _ { c } ^ { \dagger \mathbf { k } + \mathbf { q } } \left[\left|u_{\mathbf{k}, i}^{\circ}\right\rangle\right.\right. \\
& \left.\left.+\sum_{s m n}{ }^{0} I_{m n}^{s, \mathbf{q}}\left|\beta_{m}^{s, \mathbf{k}+\mathbf{q}}\right\rangle\left\langle\beta_{m}^{s} \mid \psi_{\mathbf{k}, i}^{\circ}\right\rangle\right]\right\} .
\end{aligned}
$$

If the exchange-correlation kernel is adiabatic, $\hat{v}_{\mathrm{Hxc}, \mathbf{q}}^{\prime}(\omega)$ and ${ }^{3} I_{m n}^{s, \mathbf{q}}(\omega)$ depend on $\omega$ only implicitly through the $q_{\mathbf{q}}$ batch. Actually, Eq. (16) can be rewritten in terms of $q_{\mathbf{q}}$ as

$$
\begin{aligned}
& n_{\mathbf{q}}^{\prime}(\mathbf{r}, \omega)=4 \sum_{\mathbf{k}, i}\left\{\left\langle u_{\mathbf{k}, i}^{\circ} \mid \mathbf{r}\right\rangle\left\langle\mathbf{r} \mid q_{\mathbf{k}+\mathbf{q}, i}\right\rangle\right. \\
& \left.+\sum_{s m n} \tilde{Q}_{m n}^{\gamma(s), \mathbf{q}}\left(\mathbf{r}-\boldsymbol{\tau}_{s}\right)\left\langle\psi_{\mathbf{k}, i}^{\circ} \mid \beta_{m}^{s}\right\rangle C_{n}^{s, \mathbf{k}+\mathbf{q}, i}(\omega)\right\}
\end{aligned}
$$

where

$$
C_{n}^{s, \mathbf{k}+\mathbf{q}, i}(\omega)=\int d^{3} r \beta_{n}^{s *}\left(\mathbf{r}-\boldsymbol{\tau}_{s}\right) e^{i(\mathbf{k}+\mathbf{q}) \cdot \mathbf{r}}\left\langle\mathbf{r} \mid q_{\mathbf{k}+\mathbf{q}, i}\right\rangle .
$$

In this case, the susceptibility $\chi(\mathbf{q}, \mathbf{q}, \omega)$ is given by the following expression [27, 28]:

$$
\chi(\mathbf{q}, \mathbf{q}, \omega)=\left\langle\left(y_{\mathbf{q}}, 0\right) \mid\left(\begin{array}{cc}
\omega & -\hat{\mathcal{D}}_{\mathbf{q}} \\
-\hat{\mathcal{D}}_{\mathbf{q}}-\hat{\mathcal{K}}_{\mathbf{q}} & \omega
\end{array}\right)^{-1}\left(\begin{array}{c}
0 \\
y_{\mathbf{q}}
\end{array}\right)\right\rangle,
$$

and Eq. (34) can be solved using the Lanczos recursive algorithm [17, 18, 21, 26-29, 36] identical to that used for the NC-PP case for any desired range and number of frequencies at the same computational cost, irrespective of the number of frequencies. This is important when semicore states need to be included in the calculation and treated as valence states, in order to extend the frequency range on which the EEL spectrum is computed. We point out that in gold changes brought in by the introduction of semicore states in the valence show up above $15 \mathrm{eV}$ (see appendix B).

We also note that the multiplication by $\hat{S}_{\mathbf{k}+\mathbf{q}}^{-1}$ is crucial to obtain an expression of the Liouvillian that has a form similar to the NC-PP case and can be solved by the Lanczos method [23]. In other words, we use $\hat{S}_{\mathbf{k}+\mathbf{q}}^{-1}$ in order to have equations in which the frequency $\omega$ enters as a parameter, such that we can tridiagonalize the 
resulting Liouvillian (defined by Eqs. (35) and (36)) independently on the value of the frequency. Ultimately, in the postprocessing step, the tridiagonal matrix is used to solve linear systems for various values of frequency $\omega$ at a negligible computational cost.

\section{APPLICATIONS: EEL SPECTRA OF BULK AU}

In the present work we present results obtained using the LL method and US-PPs. The EEL spectra of bulk $\mathrm{Au}$ at vanishing $\mathbf{q}$ are presented, as well as the peak dispersion, and the origin of the peaks is discussed.

\section{A. Implementations}

The LL approach to EELS [26-28] has been implemented in the turboEELS code [29], which is being distributed with the QUANTUM ESPRESSO suite [41]. The ultrasoft capabilities introduced in this paper are available as the release 6.3 of QUANTUM ESPRESSO (stable version of turboEELS with US-PPs). The Sternheimer approach to TDDFPT has been implemented in the private branch of the Quantum ESPRESSO project contained in the Thermo_pw code [42]. Sternheimer functionalities, for both norm-conserving and ultrasoft pseudopotentials, will be made available with the next version of the official distribution of QUANTUM ESPRESSO [41].

In a previous work $[16,31]$, we compared the results and performance of the LL and Sternheimer approaches using NC-PPs. We showed that the two approaches yield the same results and demonstrated that calculations performed using the LL method require a CPU time smaller than for calculations performed using Sternheimer's approach when a given frequency range needs to be considered $[16,31]$. In the present work, we have verified that also in the US-PP case both codes and both approaches give the same results. Performance turned out to be in favor of the LL method and the gain comparable to the case of NC-PPs. We report results at vanishing $\mathbf{q}$, and point out that the method is well suited for finite $\mathbf{q}$ by showing the peak dispersion for gold.

\section{B. Computational details}

The results reported in the present work were obtained in the scalar-relativistic approximation, within the local-density approximation (LDA) for the exchangecorrelation energy, and using US-PPs for $\mathrm{Au}$ with 11 $\left(5 d^{10} 6 s^{1}\right)$ or $19\left(5 s^{2} 5 p^{6} 5 d^{10} 6 s^{1}\right)$ electrons included in the valence region [43]. Two projectors have been used for each of the $s, p$ and $d$ channels [44]. For the 19 electron PP, reference energies for the $s$ angular momentum consisted of the $5 s$ and $6 s$ energy levels (resp. $5 p$ and
$6 p$ energies for the $p$ angular momentum) [44].We have also used the generalized gradient approximation (GGA) with the PBE functional $[45,46]$ in order to have a close comparison with the PBE-based calculations of ref. 47.

The experimental lattice parameter at room temperature, $a_{0}=4.08 \AA$, was used [48]. A kinetic energy cutoff of 20 Ry was used for the plane-wave expansion of the pseudowave functions for the US-PP with 11 electrons (160 Ry for the charge density) and 55 Ry was used for the plane-wave expansion of the pseudowave functions for the NC-PP and US-PP with 19 electrons (220 Ry for the charge density). Note that the unusually small kinetic energy cut-off for the US-PP of Au with 11 electrons is sufficient to converge the reported spectrum [49], but might be too low for the computation of other physical properties such as phonon frequencies or elastic constants. A $\mathbf{k}$ point mesh containing $32 \times 32 \times 32$ special Monkhorst-Pack points was used for integrations over the BZ. The Methfessel-Paxton smearing was set to $2 \mathrm{mRy}$.

Spectra for a very small value of the transferred momentum have been obtained at $|\mathbf{q}| \approx 0.03 \AA^{-1}$ in the $\Gamma-X$ direction of the BZ. The dispersion has been computed along this direction. In order to obtain a converged EEL spectrum, 6000 Lanczos iterations have been computed and the Lanczos coefficients have been extrapolated to 30000 iterations with the constant extrapolation method [29]. The frequencies used to compute the EEL spectrum of bulk Au have an imaginary part of $10 \mathrm{mRy}$ that yields a Lorentzian broadening of the peaks.

To substantiate our analysis, calculations in the random phase approximation (RPA) neglecting local field effects have been performed with the SIMPLE code at $\mathbf{q}=\mathbf{0}$ exactly [50], with the 19-electron norm-conserving pseudopotential of ref. 51. We detail the calculations made with the SIMPLE code. Both the full response and the contribution of only interband transitions (ITs) have been obtained. The bulk dielectric function $\epsilon_{B}$ at $\mathbf{q}=\mathbf{0}$ has been obtained by summing vertical $(\mathbf{q}=\mathbf{0})$ transitions, i.e. summing matrix elements of the dipolar operator between an occupied state and an empty one [50]. The intraband contribution has been added separately [50]. The loss function has then been obtained as:

$$
-\operatorname{Im}\left(\epsilon_{B}^{-1}\right)=\frac{\operatorname{Im}\left(\epsilon_{B}\right)}{\operatorname{Re}\left(\epsilon_{B}\right)^{2}+\operatorname{Im}\left(\epsilon_{B}\right)^{2}},
$$

where $\epsilon_{B}$ is the dielectric function of the bulk material without local fields effects, either containing both interband and intraband contributions, or being restricted to ITs.

Finally, in order to identify plasmon peak positions, we present the loss function of two toy models computed with LL using modified pseudopotentials: a just 1-electron $\mathrm{PP}$ where $5 d$ electrons are frozen in the core and a 10-electron PP from which the $6 s$ electron was removed. These pseudopotentials were NC-PPs and designed by us by reducing the number of electrons in the generation of the 11-electron NC-PP of ref. 52 . 
The data used to produce the results of this work is available on the Materials Cloud Archive [53].

\section{Benchmark of the Liouville-Lanczos implementation with US-PPs}

In the present section we present a validation of our implementation of the LL approach with US-PPs. The EEL spectrum obtained in TDDFPT-GGA with the 19electron US-PP developed in the present work (solid blue line), closely agree with our results obtained with the 19electron NC-PP of ref. 51 (dashed black line), and compare well with a previous work [47], where the loss function was obtained through the solution of the Dyson-like equation [5] with an all-electron full-potential linearized augmented plane-wave (FP-LAPW) method (red dashed line).

The overall remarkable agreement between the EEL spectrum obtained using US-PP with another calculation (fig. 1) on a wide energy range confirms the correctness of the implementation of the LL approach with US-PPs. It is important to note though that special care must be given to the selection of US-PPs with high accuracy and transferability if one is interested in computing EEL spectra on a wide energy range, as done in the present study.

The implementation of the LL approach with US-PPs can allow to achieve better performance with respect to NC-PPs thanks to the reduction of the cutoff and hence reduction of the CPU time. This is though element dependent. Unfortunately in Au this cannot be seen because the same value of the cutoff is needed for both types of PPs (see Sec. III B), however calculations of plasmons in other elements, for which US-PPs are in bigger contrast with NC-PPs due to the hardness of the latter, can benefit more from our implementation of the LL approach with US-PPs. More specifically, the lower the cutoff value, the smaller the number of Lanczos iterations necessary to reach convergence of the EEL spectrum: this is a property of the LL approach [18]. Moreover, not only the number of Lanczos iterations can be decreased, but also the cost of each iteration will be reduced, which overall allows us to lower the CPU time requirements despite the fact that some extra-computations are needed because of the presence of the US-PP-related terms. As a matter of fact, the LL approach with US-PPs is widely used for the optical absorption spectroscopy of molecules $[18,36]$.

\section{EEL spectra of bulk Au}

In fig. 2 we present the loss functions of bulk Au obtained in TDDFPT-LDA, with US-PPs having 19 and 11 electrons in the valence region (resp. solid-blue and dashed-black lines), and compare them with the loss function obtained in the reflection-EELS (REELS) ex-

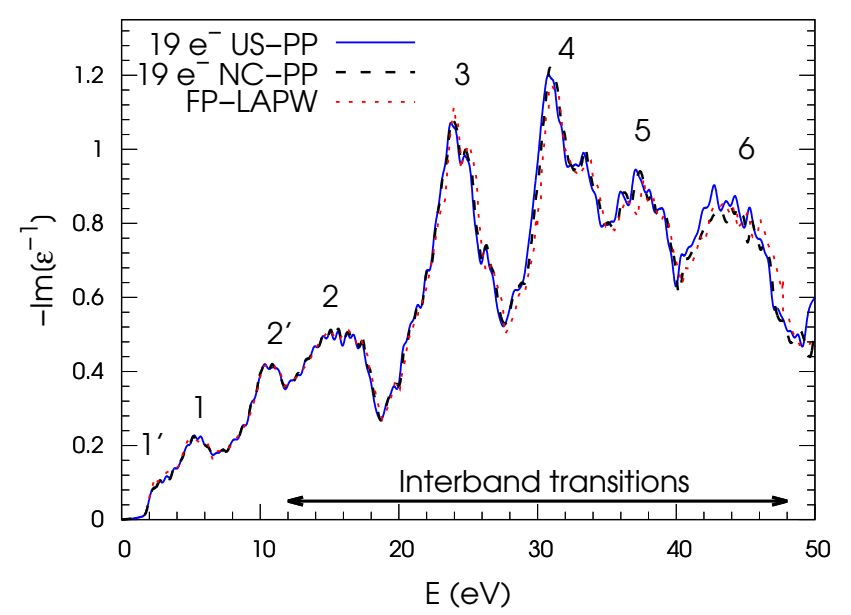

FIG. 1. EEL spectrum of bulk $\mathrm{Au}$ computed with 19 electrons in the valence region, with ultrasoft and normconserving pseudopotentials, for $|\mathbf{q}| \approx 0.03 \AA^{-1}$ in the [100] direction. Previous FP-LAPW data [47] is reported for $\mathbf{q}=0$. Peaks are labeled according to ref. 47 and we have added the label $1^{\prime}$ and $2^{\prime}$. The horizontal arrow marks the frequency domain of pure interband transitions. Computations performed in TDDFPT-GGA.

periment of ref. 54 (solid black line). We note that the difference between the EEL spectra computed with the same parameterization of US-PP but different functionals, GGA and LDA, at the same lattice parameter, look very similar (resp. figs. 1 and 2).

Also we stress that according to our findings the 19 electrons US-PP must be used on the extended energy range (up to $50 \mathrm{eV}$ in this work), while the 11 electrons US-PP is suitable for studies up to $15 \mathrm{eV}$ only (see appendix $\mathrm{B}$ for more discussion about the semicore $5 s$ and $5 p$ states), as can be seen in Fig. 2 when comparing with the experimental spectrum. Therefore, in the rest of this paper we present the results obtained with the 19 electrons US-PP.

Experimental and theoretical peak positions are summarized in table I. A slight inaccuracy w.r.t. experiment comes from our neglect of spin-orbit coupling. Peak positions obtained with the 19-electron US-PP are in good agreement with ref. 47 , with differences between $-0.6 \mathrm{eV}$ and $0.2 \mathrm{eV}$. With regard to experiment [54], peaks 1 and 2 show differences of $-0.9 \mathrm{eV}$ and $-0.8 \mathrm{eV}$, respectively, while in comparison with the experimental results of ref. 55, peaks 1 and 2 show differences of $-0.8 \mathrm{eV}$ and $-0.3 \mathrm{eV}$, respectively.

Part of the inaccuracy comes from the description of $5 d$ bands of $\mathrm{Au}$ in LDA $[47,56]$ that leads to the redshift of the interband transition onset by approximately $0.5 \mathrm{eV}$. As can be seen from table I, the redshift was only partially corrected by the approximate $G W$ calculation of ref. 47 (table I).

Finally, the contribution $1^{\prime}$ cannot be singled out as a peak, in contrast with experiment (fig. 2). This point is further discussed below. 


\begin{tabular}{|c|c|c|c|c|c|c|c|}
\hline \multirow{3}{*}{$\begin{array}{l}\text { peak } \\
\#\end{array}$} & \multicolumn{2}{|r|}{ This work } & \multicolumn{3}{|r|}{ Ref. 47} & \multicolumn{2}{|c|}{ Expt. 54 Expt. 55} \\
\hline & $\omega(\mathrm{eV})$ & Origin & $\omega(\mathrm{eV})$ & & Origin & & $\omega(\mathrm{eV})$ \\
\hline & $19 \mathrm{e}$ US-PP & & FP-LAPW & $G W$ & & & REELS \\
\hline $1^{\prime}$ & 2.2 & Mixed excitation & 2.2 & 2.65 & Weak plasmon-like peak & 3.25 & 2.5 \\
\hline 1 & 5.1 & $5 d$ plasmon & 5.3 & 5.6 & Plasmon-type & 6.0 & 5.9 \\
\hline $2^{\prime}$ & 10.2 & Mainly-6s plasmon & 10.5 & 11.0 & & $10.2^{\mathrm{a}}$ & $11.9^{\mathrm{a}}$ \\
\hline 2 & 15.5 & IT & 15.4 & - & IT & 16.3 & 15.8 \\
\hline 3 & 23.8 & IT & 24.0 & - & IT & 23.6 & 23.6 \\
\hline 4 & 30.8 & IT & 31.1 & - & IT & 31.2 & 31.5 \\
\hline 5 & 36.9 & IT & 37.5 & - & IT & - & 39.5 \\
\hline 6 & 43.5 & IT & 43.3 & - & IT & - & 44.0 \\
\hline
\end{tabular}

${ }^{\text {a }}$ Appears as a shoulder in the spectrum.

TABLE I. Bulk Au. Energy of the peak positions in the loss function shown in fig. 2 for the 19-electron US-PP in LDA. Peaks are labeled according to ref. 47 and we have added the labels $1^{\prime}, 2^{\prime}$. "IT" stands for "interband transition" and, by "mixed excitation", we mean IT modified by the intraband (Drude) contribution. ITs are present everywhere between $2.2 \mathrm{eV}$ and 50 $\mathrm{eV}$ as a background contribution.

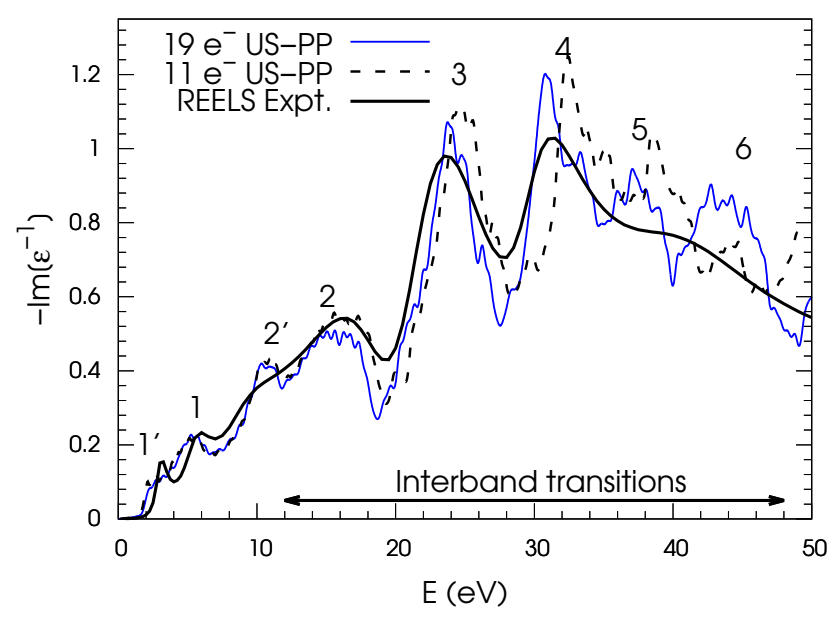

FIG. 2. EEL spectrum of bulk Au computed with US-PP and 11 valence electrons (black dashed line) or 19 valence electrons (blue solid line) for $|\mathbf{q}| \approx 0.03 \AA^{-1}$ in the [100] direction. Solid black line: from REELS experiment [54] at $\mathbf{q}=\mathbf{0}$. Peak labels as in fig. 1. Computations performed in TDDFPT-LDA.

\section{E. Origin and dispersion of the peaks}

\section{Interband transitions}

We found the presence of ITs between $2.2 \mathrm{eV}$ and $50 \mathrm{eV}$, i.e. the highest energy for which the EEL spectrum has been computed (fig. 3, center panel, dotted line). Interband transitions are characterized in gold by a very weak dispersion above $12 \mathrm{eV}$, and peaks 2-6 are attributed to "pure" interband transitions (fig. 5, bottom panel). Below $12 \mathrm{eV}$, ITs are dispersing, which is the fingerprint of their mixing with plasmon excitations, as will be discussed in section IIIE 3 (fig. 5, top panel).
The attribution of peaks beyond $15 \mathrm{eV}$ is in agreement with ref. 47.

\section{Plasmon excitations}

The energy of a collective excitation like a plasmon cannot be deduced directly from the band structure. Bulk plasmon energies are coming from the zeroes of the real part of the dielectric function, with a positive slope of the real part of $\epsilon$. The crossing of the zero energy axis strictly implies the existence of a self-sustaining oscillation.

In gold, there are two such oscillation frequencies, as the real part of the dielectric function crosses the zero energy axis at $4.8 \mathrm{eV}$ and at $10.05 \mathrm{eV}$ (fig. 3, bottom panel). Therefore peaks 1 and $2^{\prime}$ of the loss function are unambiguously attributed to plasmons at $5.1 \mathrm{eV}$ and $10.2 \mathrm{eV}$, respectively (fig. 3, top panel). The difference in frequency between the zeroes of the real part, and the positions of the two plasmon peaks, is explained below in the present section. The attribution of the peak at $5.1 \mathrm{eV}$ to a plasmonic excitation is in agreement with ref. 47 .

On the other hand, peak $2^{\prime}$, which is also found in the calculated spectrum of ref. 47 (table I), has not been discussed so far. Thanks to the two toy-model PP calculations with only $6 s$ electrons and with only $5 d$ electrons, we unambiguously conclude that the plasmon peak $2^{\prime}$ is coming mainly from the collective excitation of $6 s$ electrons. Indeed, in fig. 4, the spectrum computed with a toy-model containing only the $6 s$ electron is represented by a single plasmon at $10.05 \mathrm{eV}$ (red dotted line). There is hardly any peak in the calculation with only $5 d$ electrons and no $6 s$ ones, while all of the calculations with more than 10 electrons consistently show a peak at $10.2 \mathrm{eV}$.

On the other hand, peak 1 is attributed to a plasmon 

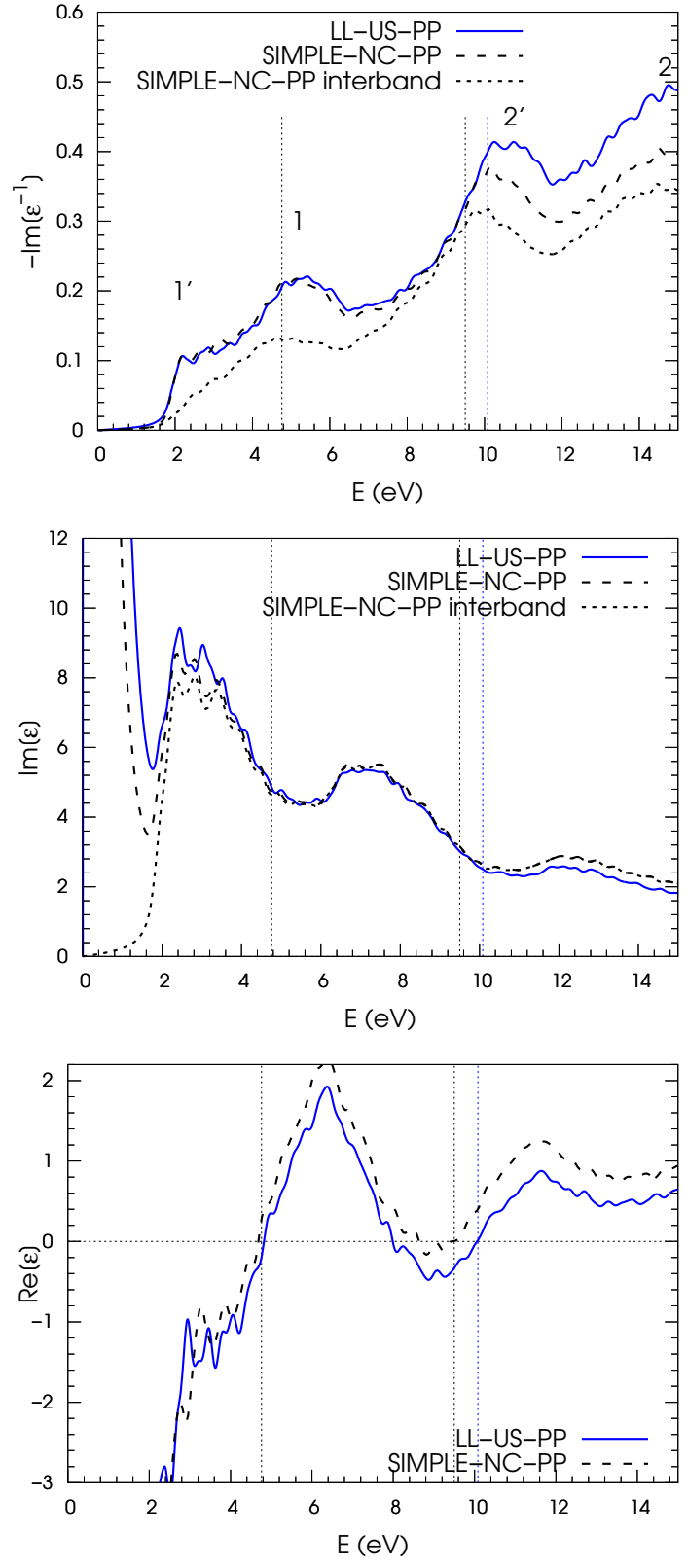

FIG. 3. Dielectric function (DF) and loss function (LF) of bulk Au with 19 electron PPs. Real part (bottom panel) and imaginary part (center panel) of the DF and LF (top panel) up to $15 \mathrm{eV}$ for $|\mathbf{q}| \approx 0.03 \AA^{-1}$ in the [100] direction. Solid lines: Liouville-Lanczos's (LL) method as implemented in the turboEELS code. Dashed lines: Simple code at $\mathbf{q}=\mathbf{0}$ with both interband and intraband contributions. Dotted line: interband contributions only to the imaginary part of the DF at $\mathbf{q}=\mathbf{0}$. The vertical bars show the zeroes of the real part (all panels). Slights differences that are observed in the imaginary part and real part of the DF below $10 \mathrm{eV}$ when comparing the calculation at $\mathbf{q}=\mathbf{0}$ and the calculation at $|\mathbf{q}| \approx 0.03 \AA^{-1}$ comes from the diverging behavior of the intraband contribution at $\mathbf{q}=\mathbf{0}$. Differences that are observed above $10 \mathrm{eV}$ are due to the neglect of local fields in the calculation with the SIMPLE code at $\mathbf{q}=\mathbf{0}$. Computations in TDDFPT-LDA.

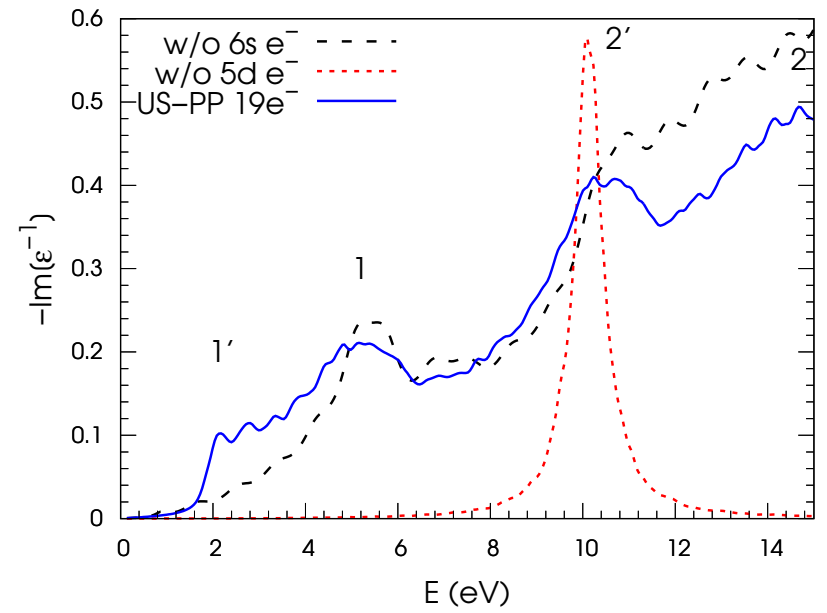

FIG. 4. Toy-model EEL spectra computed using modified NC-PP pseudopotentials: without the $6 s$ electron (black dashed line) or without $5 d$ electrons (red dashed line). They are compared to the EEL spectrum of the 19-electron USPP that contains both $5 d$ and $6 s$ electrons (blue solid line, same as in fig. 2). In the legend, "w/o" stands for without. Results computed using the LL method. Computation performed within TDDFPT-LDA.

oscillation from $5 d$ electrons. Indeed, in fig. 4 , the spectrum computed with the toy model PP without the $6 s$ electron, containing only $5 d$ electrons, has a well pronounced peak at $5.1 \mathrm{eV}$ (black dashed line), in close-toperfect agreement with the plasmon positions reported in ref. 47 (table I). Thus, electrons in gold behave as two quasi-separate electron gasses, each one oscillating with its own frequency.

However, as seen above, in our calculations, the two plasmons are intimately influenced by the interband transitions contained in $\operatorname{Im}(\epsilon)$ around the respective plasmon frequencies. This is the reason why the zeros of the real part of the dielectric function (resp. $4.8 \mathrm{eV}$ and at $10.05 \mathrm{eV}$ ) and the main peaks of the energy loss function $(5.1 \mathrm{eV}$ and $10.2 \mathrm{eV})$ do not exactly coincide: the imaginary part is not minimal at the position of the zeros of the real part in the calculations, and the plasmon positions are blue-shifted by $0.3 \mathrm{eV}$ and approximately $0.15 \mathrm{eV}$ w.r.t. the zeros of the real part of the dielectric function. We point out that while crystal local fields have no effect on the zero energy crossing at $4.8 \mathrm{eV}$ coming from the $5 d$ electron gas (fig. 3, bottom panel, dotted vertical line on the left-hand side), they have an important effect at the $10.05 \mathrm{eV}$ (fig. 3, bottom panel, black and blue and dotted vertical lines on the right-hand side) [57]. We also note that in the experimental data reported around $10 \mathrm{eV}$, the real part of the dielectric function is found to be positive [58].

Finally, inspection of our calculations does not allow us to attribute a bulk plasmonic origin to the peak at $3.25 \mathrm{eV}$ [54] or $2.5 \mathrm{eV}$ [55] observed in the REELS experiments (table I). This point is discussed in more detail in the next section. 


\section{Mixed excitations}

In this section we discuss the remaining peaks in the EEL spectra of bulk Au. There is no clear peak that can be singled out near $2 \mathrm{eV}$ in the loss function (contribution $1^{\prime}$, fig. 3 , top panel) nor any zero of the real part of the dielectric function near $2 \mathrm{eV}$ in the scalar-relativistic calculation (bottom panel). Thus contributions in the loss function between $\approx 2$ and $\approx 4 \mathrm{eV}$ are due to $5 d \rightarrow 6 s$ interband contributions. We note however that, in this energy interval, the interband contributions are modified by the intraband component of the excitation coming from the presence of a plasmon at $4.8 \mathrm{eV}$ in the real part of the dielectric function. This can be numerically checked by inserting in eq. (41) $\operatorname{Re}\left(\epsilon_{B}\right)$ of the total dielectric function (both intraband and interband) and $\operatorname{Im}\left(\epsilon_{B}\right)$ containing only the interband contribution (not shown). Thus contribution $1^{\prime}$ near $2.2 \mathrm{eV}$ is attributed to mixed excitations, and has no plasmonic origin (table I).

On the other hand, the interpretation of the contribution near $2.2 \mathrm{eV}$ in previous works[47] was made in analogy with the plasmon in silver. In silver, there is a plasmon at $3.8 \mathrm{eV}$ whose position is due to the shift of the mainly- $s$ plasmon at $9.7 \mathrm{eV}$, caused by the presence of interband transitions [47]. By analogy, in gold, it was thought that a very weak plasmon-like peak was developing at $2.65 \mathrm{eV}$ when calculations were performed with methods beyond DFT, e.g. with the approximate $G W$ calculations (ref. 47, supplemental material). In gold however, we find the well-defined $s$ plasmon at $10.2 \mathrm{eV}$ (see previous section), and a related peak is also found in the $G W$ calculations at $11.0 \mathrm{eV}$ (table I). Consequently, the contribution near $2.2 \mathrm{eV}$, in bulk gold, is probably solely made of a wealth of $5 d \rightarrow 6 s$ interband contributions.

Moreover, it should be noted that, from the experimental side, extra complications come from the fact that, at a similar energy, several contributions show up [55]. In particular, the deconvolution of the bulk and surface contributions from the experimental total spectra is very sensitive to many details. This makes it hard to determine precisely the exact position of the contribution $1^{\prime}$ (table I, last two columns on the right-hand side).

Finally, the dispersion of the contribution $1^{\prime}$ is reported in fig. 5 (top panel). Indeed, as the loss function due to interband transitions is modified by the intraband contribution to the real part of the dielectric function, it shows some dispersion. This is the case for the contribution $1^{\prime}$ in gold, as well as for the peak at $5 \mathrm{eV}$ in bulk bismuth [59]. This is general to materials in which there are interband transitions below the (not too far) plasmon energy: interband transitions then show some dispersion.

\section{CONCLUSION}

In conclusion, in the present paper we have demonstrated how the Liouville-Lanczos and the Sternheimer
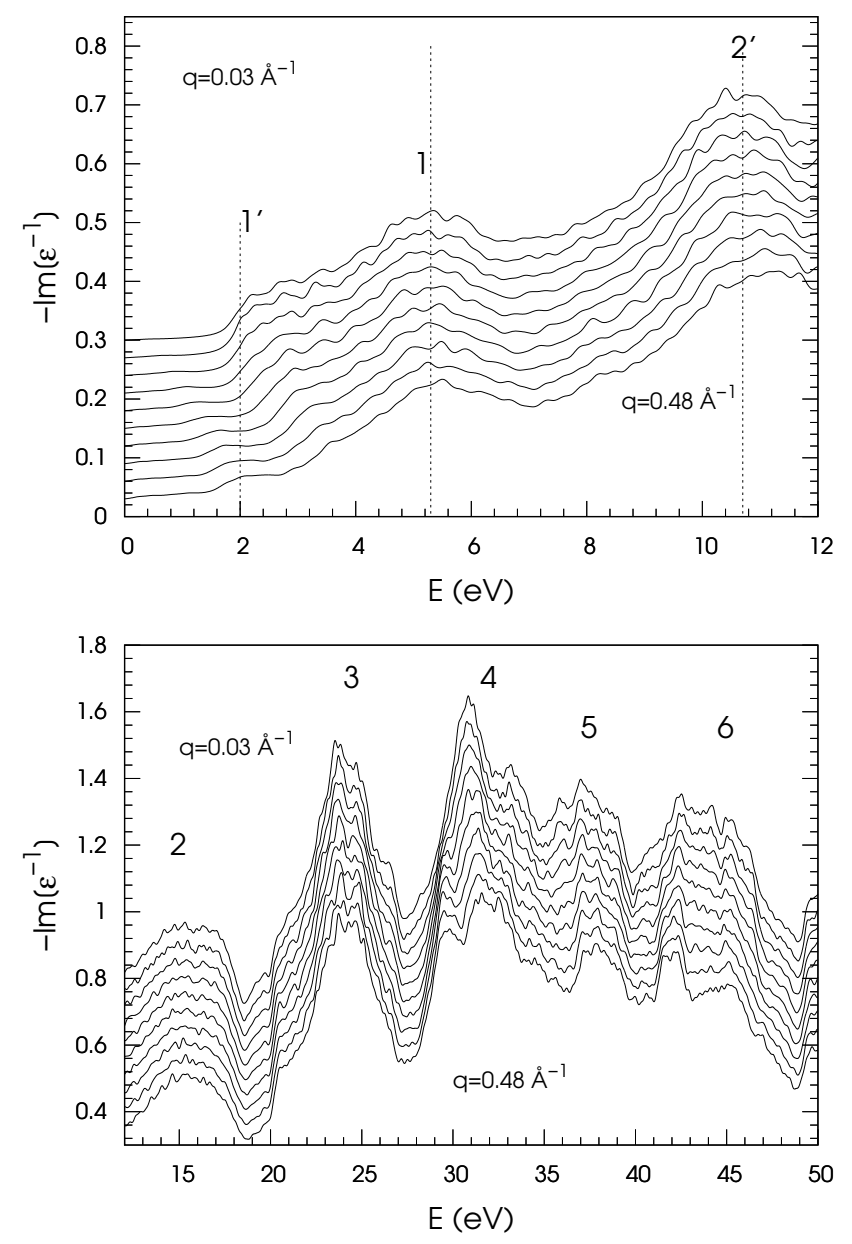

FIG. 5. Loss function of bulk Au with the 19 electron US-PP. Loss function as a function of energy and transferred momentum $q$ in the [100] direction (black solid lines). Energy below $12 \mathrm{eV}$ (top) and above $12 \mathrm{eV}$ (bottom panel). The thin dotted lines in the top panel are guides for the eyes to highlight the peak dispersion. Results computed with the LL method within TDDFPT-LDA.

approaches to the TDDFPT calculations of electron energy loss spectra and inelastic X-ray scattering crosssections can be generalized to Vanderbilt's US-PPs. We have shown, on the example of bulk Au, that results obtained with the LL method and US-PPs agree with other TDDFT studies.

We have analyzed in detail the origin of various peaks in the EEL spectra of bulk Au. We have found that the signature of plasmons in EEL spectra of bulk Au revealed by our study shows the richness of the physics underlying the various contributions to the density fluctuation in gold. In particular, we have attributed peaks at $5.1 \mathrm{eV}$ and $10.2 \mathrm{eV}$ to plasmon excitations coming from $5 d$ and $6 s$ electron gasses, respectively. We have defined as a mixed excitation a contribution from interband transitions that lies below the plasmon frequencies and is modified by the real part of the intraband contribution, and given a way to characterize numerically a 
mixed excitation in the calculations. We have then identified the contributions between $\approx 2.2 \mathrm{eV}$ and $\approx 4 \mathrm{eV}$ as mixed excitations. We have concluded that alone, contributions of bulk Au cannot explain the presence of the well-defined peak at low energy observed in the REELS experiments. Finally we have shown the dispersion of plasmons and mixed excitation, and the very weak dispersion of pure interband transitions at energies above the plasmon frequencies.

\section{ACKNOWLEDGMENTS}

Results have been obtained with the turboEELS and SIMPLE codes of the QUANTUM ESPRESSO [41] project, and with the Thermo_pw [42] package. Computer time has been granted by the Partnership for Advanced Computing in Europe (PRACE Project No. 2010PA3750), by the national centers GENCI-CINES and GENCI-TGCC (Project 2210), and by École Polytechnique through the LLR-LSI project.

O.M. acknowledges support from the doctoral school INTERFACES of École Polytechnique, and from the EUMaX project, and fruitful discussions with M. Raynaud at an early stage of the project. This work was partly funded by the EU Commission through the MaX Centre of Excellence for Supercomputing Applications, grants no. 676598 and 824143 . I.T. acknowledges support from the Swiss National Science Foundation (SNSF), through grant 200021-179138, and its National Centre of Competence in Research (NCCR) MARVEL. ADC acknowledges also support from the SISSA ITCS and its Linux cluster.

\section{Appendix A: Inverse overlap operator for periodic solids}

In the US-PPs case, the inverse of the overlap operator $\hat{S}$ needs to be introduced in the Liouville-Lanczos + NC-PPs algorithm of the TDDFPT equations. It was introduced in ref. 36 as:

$$
\hat{S}^{-1}=1+\sum_{I J, m n} \lambda_{m n}^{I J}\left|\beta_{m}^{I}\right\rangle\left\langle\beta_{n}^{J}\right|
$$

where the sum over $I$ and $J$ runs on the atoms of the system and the sum over $m$ and $n$ runs over the projectors of each atom. In ref. 36, the coefficients $\lambda_{m n}^{I J}$ are obtained from the condition $\hat{S} \hat{S}^{-1}=1$ which gives the linear systems:

$$
\sum_{I m}\left(\delta_{r m} \delta_{N I}+\sum_{i} q_{r i}^{N} B_{i m}^{N I}\right) \lambda_{m n}^{I J}=-q_{r n}^{N} \delta_{N J},
$$

where $B_{i m}^{N I}=\left\langle\beta_{i}^{N} \mid \beta_{m}^{I}\right\rangle$ and $\delta_{N I}$ is the Kroenecker symbol. There are $N_{B}$ linear systems (for each $J$ and $n$ ) of size $N_{B} \times N_{B}$, where $N_{B}$ is the number of projectors in the system.
In a periodic solid we can write $I=\{l, s\}, J=\left\{l^{\prime}, s^{\prime}\right\}$ and $N=\left\{l^{\prime \prime}, s^{\prime \prime}\right\}$ and use periodicity to remove the dependence on the unit cell index, obtaining in this way $N_{b}$ linear systems of dimension $N_{b} \times N_{b}$, where $N_{b}$ is the number of projectors in one unit cell. Clearly different systems will be obtained for each $\mathbf{k}$-point in the Brillouin zone. To compute the action of the operator $\hat{S}^{-1}$ on a Bloch function $\psi_{\mathbf{k}, i}(\mathbf{r})$ it is convenient to define the coefficients:

$$
\lambda_{m n}^{\mathbf{k}, s s^{\prime}}=\sum_{l^{\prime}} e^{-i \mathbf{k} \cdot \mathbf{R}_{l}} \lambda_{m n}^{l s, l^{\prime} s^{\prime}} e^{i \mathbf{k} \cdot \mathbf{R}_{l^{\prime}}}
$$

where $\lambda_{m n}^{\mathbf{k}, s s^{\prime}}$ are independent from $l$ since $\lambda_{m n}^{l s, l^{\prime} s^{\prime}}$ depend on $\mathbf{R}_{l}-\mathbf{R}_{l^{\prime}}$. Inserting this definition in the expression of $\hat{S}^{-1}$ we find:

$$
\begin{aligned}
& \sum_{I J, m n} \lambda_{m n}^{I J}\left|\beta_{m}^{I}\right\rangle\left\langle\beta_{n}^{J} \mid \psi_{\mathbf{k}, v}\right\rangle= \\
& \sum_{s s^{\prime}, m n} \lambda_{m n}^{\mathbf{k}, s s^{\prime}} \sum_{l}\left|\beta_{m}^{I}\right\rangle e^{i \mathbf{k} \mathbf{R}_{l}}\left\langle\beta_{n}^{s^{\prime}} \mid \psi_{\mathbf{k}, v}\right\rangle .
\end{aligned}
$$

For each $\mathbf{k}$, the $N_{b}^{2}$ coefficients $\lambda_{m n}^{\mathbf{k}, s s^{\prime}}$, are solutions of the $N_{b}$ linear systems (for each $s^{\prime}$ and $n$ ):

$$
\sum_{s m}\left(\delta_{r m} \delta_{s^{\prime \prime} s}+\sum_{i} q_{r i}^{s^{\prime \prime}} B_{i m}^{\mathbf{k}, s^{\prime \prime} s}\right) \lambda_{m n}^{\mathbf{k}, s s^{\prime}}=-q_{r n}^{s^{\prime \prime}} \delta_{s^{\prime \prime} s^{\prime}}
$$

where

$$
B_{i m}^{\mathbf{k}, s^{\prime \prime} s}=\sum_{l} e^{i \mathbf{k} \cdot \mathbf{R}_{l}} \int d^{3} r \beta_{i}^{\gamma\left(s^{\prime \prime}\right) *}\left(\mathbf{r}-\boldsymbol{\tau}_{s^{\prime \prime}}\right) \beta_{m}^{\gamma(s)}\left(\mathbf{r}-\mathbf{R}_{l}-\boldsymbol{\tau}_{s}\right) .
$$

which can be obtained by multiplying Eq. A2 by $e^{i \mathbf{k} \cdot \mathbf{R}_{l^{\prime}}}$ and adding on $l^{\prime}$.

Finally, the operator $\hat{S}_{\mathbf{k}}^{-1}$ that appear in Eqs. (35), (36), and (37) can be obtained from the relationship:

$$
\hat{S}^{-1} \psi_{\mathbf{k}, i}(\mathbf{r})=e^{i \mathbf{k} \cdot \mathbf{r}} \hat{S}_{\mathbf{k}}^{-1} u_{\mathbf{k}, i}(\mathbf{r}),
$$

and can be written as:

$$
\hat{S}_{\mathbf{k}}^{-1}=1+\sum_{s s^{\prime}, m n} \lambda_{m n}^{\mathbf{k}, s s^{\prime}}\left|\beta_{m}^{s, \mathbf{k}}\right\rangle\left\langle\beta_{n}^{s^{\prime}, \mathbf{k}}\right|
$$

We benchmarked the implementation of the operator $\hat{S}_{\mathbf{k}}^{-1}$ in the LL method by comparing the final results with the results obtained using the method based on the solution of the Sternheimer equations, since the latter does not require $\hat{S}_{\mathbf{k}}^{-1}$.

\section{Appendix B: Effect of the semicore states}

In the present appendix we discuss the effect of semicore $5 s$ and $5 p$ states on the EEL spectrum of bulk Au.

This is an important aspect, because in many studies semicore states are frozen in the core, and hence it is important to clarify in which energy range this approximation gives reliable results. In fact, in the case of Bi it was 
shown that by freezing the $5 d$ semicore states in the core region, the plasmon peak position is strongly affected and the high-energy part of the spectrum is completely missing [59]. In a previous study of the electronic bandstructure of gold, inclusion of the semicore states was shown to have practically no effect on the DFT-level, but to be very important in the $G W$ calculations [56]. Here we determine the energy range on which the EEL spectra are accurate with $5 s$ and $5 p$ semicore states frozen in the core in the case of gold, and also we highlight at which energies the effect of $5 s$ and $5 p$ in the valence region is crucial.

In this work we used two types of US-PPs: the 11 electrons case (with $5 s$ and $5 p$ semicore states frozen in the core) and 19 electrons case (with $5 s$ and $5 p$ semicore states included in the valence region of the electronic configuration). We find that the US-PPs with 11 and 19 electrons are in close-to-perfect agreement with each other up to $15 \mathrm{eV}$, i.e. for contribution $1^{\prime}$ and peaks 1 and $2^{\prime}$ (see Fig. 2 and Table II). This validates the use of the US-PP with 11 electrons to study low energy excitations in large systems made of gold atoms, and confirms an anterior work [60]. However, for energies above $15 \mathrm{eV}$ there are significant deviations in the peak positions and in their intensities (peaks 2-6).

Moreover, the origins of the peaks are also left unchanged: at low energy all is exactly the same, while at higher energies all peaks come from the interband transitions (with missing interband contributions in the 11 electrons case due to missing initial states ( $5 s$ and $5 p$ ) which are frozen in the core).

Therefore, we conclude that the 11 electrons US-PP can be safely used to describe low energy excitations, while the 19 electrons case is absolutely needed for investigations of extended energy portions of the EEL spectra.

\begin{tabular}{lllllllll}
\hline \hline Peak \# $1^{\prime}$ & 1 & $2^{\prime}$ & 2 & 3 & 4 & 5 & 6 \\
\hline 11 elec. & 2.2 & 5.3 & 10.5 & 16.1 & 24.7 & 32.5 & 38.5 & 44.0 \\
19 elec. & 2.2 & 5.1 & 10.2 & 15.5 & 23.8 & 30.8 & 36.9 & 43.5 \\
\hline \hline
\end{tabular}

TABLE II. Peak positions (in eV) in the EEL spectrum of bulk Au (see fig. 2) computed with two types of US-PPs, containing 11 electrons (without semicore states) and 19 electrons (with semicore states) in the valence.
[1] E. Runge and E.K.U. Gross, "Density-functional theory for time-dependent systems," Phys. Rev. Lett. 52, 997 (1984).

[2] E. K. U. Gross and W. Kohn, "Local density-functional theory of frequency-dependent linear response," Phys. Rev. Lett. 55, 2850 (1985).

[3] E. K. U. Gross, J. F. Dobson, and M. Petersilka, Density Functional Theory of Time-Dependent Phenomena, Topics in Current Chemistry (Springer-Verlag, Berlin, 1996).

[4] V. Olevano and L. Reining, "Excitonic effects on the silicon plasmon resonance," Phys. Rev. Lett. 86, 5962 (2001).

[5] G. Onida, L. Reining, and A. Rubio, "Electronic excitations: Density functional versus many body Green's functions approaches," Rev. Mod. Phys. 74, 601 (2002).

[6] G. Fugallo S.M.Anderson, B.S. Mendoza and F. Sottile, "Phonon dispersion in graphite: A comparison of current ab initio methods," Phys. Rev. B 100, 0452053 (2019).

[7] C. Cochi C. Vorwek, B. Aurich and C. Draxl, "BetheSalpeter equation for absorption and scattering spectroscopy: implementation in the ExcITING code," Electron. Struct. 1, 037001 (2019).

[8] J.J. Kas, J.J. Rehr, and L. Reining, "Cumulant expansion of the retarded one-electron green function," Phys. Rev. B 90, 085112 (2014).

[9] M. Guzzo, J.J. Kas, L. Sponsa, C. Giorgetti, F. Sottile, D. Pierucci, M.G. Silly, F. Sirotti, J.J. Rehr, and L. Reining, "Multiple satellites in materials with complex plasmon spectra: From graphite to graphene," Phys. Rev. B 89, 085425 (2004).

[10] J. P. Nery and P. B. Allen, "Influence of Fröhlich polaron coupling on renormalized electron bands in polar semiconductors: Results for zinc-blende GaN," Phys. Rev. B 94, 115135 (2016).
[11] J. P. Nery, P. B. Allen, G. Antonius, L. Reining, A. Miglio, and X. Gonze, "Quasiparticles and phonon satellites in spectral functions of semiconductors and insulators: Cumulants applied to the first-principles theory and the Fröhlich polaron," Phys. Rev. B 97, 115145 (2016).

[12] D.C. Yost, Y. Yao, and Y. Kanai, "Examining realtime time-dependent density functional theory nonequilibrium simulations for the calculation of electronic stopping power," Phys. Rev. B 96, 115134 (2017).

[13] Nicolas Tancogne-Dejean, Micael J. T. Oliveira, and Angel Rubio, "Self-consistent DFT $+U$ method for realspace time-dependent density functional theory calculations," Phys. Rev. B 96, 245133 (2017).

[14] Y. Miyamoto and A. Rubio, "Application of the real-time time-dependent density functional theory to excited-state dynamics of molecules and 2D materials," J. Phys. Soc. Jap. 87, 041016 (2018).

[15] S. Baroni, S. de Gironcoli, A. Dal Corso, and P. Giannozzi, "Phonons and related crystal properties from density-functional perturbation theory," Rev. Mod. Phys. 73, 515 (2001).

[16] O. Motornyi, M. Raynaud, A. Dal Corso, and N. Vast, "Simulation of electron energy loss spectra with the turboEELS and thermo_pw codes," J. Phys.: Conf. Ser. 1136, 012008 (2018).

[17] B. Walker, A. M. Saitta, R. Gebauer, and S. Baroni, "Efficient approach to time-dependent density-functional perturbation theory for optical spectroscopy," Phys. Rev. Lett. 96, 113001 (2006).

[18] D. Rocca, R. Gebauer, Y. Saad, and S. Baroni, "Turbo charging time-dependent density-functional theory with Lanczos chains," J. Chem. Phys. 128, 154105 (2008).

[19] S. Baroni and R. Gebauer, "Fundamentals of time- 
dependent density functional theory," (Springer, Berlin, 2012) Chap. 19-The Liouville-Lanczos Approach to Time-Dependent Density-Functional Perturbation Theory, pp. $375-390$.

[20] D. Rocca, R. Gebauer, F. De Angelis, M. K. Nazeeruddin, and S. Baroni, "Time-dependent density functional theory study of squaraine dye-sensitized solar cells," Chem. Phys. Lett. 475, 49 (2009).

[21] O. B. Malcioglu, R. Gebauer, D. Rocca, and S. Baroni, "TurboTDDFT - a code for the simulation of molecular spectra using the Liouville-Lanczos approach to time-dependent density-functional perturbation theory," Comput. Phys. Commun. 182, 1744 (2011).

[22] P. Ghosh and R. Gebauer, "Computational approaches to charge transfer excitations in a zinc tetraphenyl prophyrin and C-70 complex," J. Chem. Phys. 132, 104102 (2011).

[23] R. Gebauer and F. De Angelis, "A combined molecular dynamics and computational spectroscopy study of a dye-sensitized solar cell," New J. Phys. 13, 085013 (2013).

[24] D. Rocca, D. Lu, and G. Galli, "Ab initio calculations of optical absorption spectra: Solution of the BetheSalpeter equation within density matrix perturbation theory," J. Chem. Phys. 133, 164109 (2010).

[25] D. Rocca, Y. Ping, R. Gebauer, and G. Galli, "Solution of the Bethe-Salpeter equation without empty electronic states: Application to the absorption spectra of bulk systems," Phys. Rev. B 85, 045116 (2012).

[26] I. Timrov, Ab initio study of plasmons and electronphonon coupling in bismuth: From free-carrier absorption towards a new method for electron energy-loss spectroscopy, Ph.D. thesis, École Polytechnique, France (2013).

[27] I. Timrov, N. Vast, R. Gebauer, and S. Baroni, "Electron energy loss and inelastic x-ray scattering cross sections from time-dependent density-functional perturbation theory," Phys. Rev. B 88, 064301 (2014).

[28] I. Timrov, N. Vast, R. Gebauer, and S. Baroni, "Erratum: Electron energy loss and inelastic x-ray scattering cross sections from time-dependent density-functional perturbation theory [Phys. Rev. B 88, 064301 (2013)]," Phys. Rev. B 91, 139901(E) (2015).

[29] I. Timrov, N. Vast, R. Gebauer, and S. Baroni, "turboEELS-A code for the simulation of the electron energy loss and inelastic x-ray scattering spectra using the Liouville-Lanczos approach to time-dependent densityfunctional perturbation theory," Computer Physics Communications 196, 460-469 (2015).

[30] T. Gorni, I. Timrov, and S. Baroni, "Spin dynamics from time-dependent density functional perturbation theory," The European Physical Journal B 91, 249 (2018).

[31] O. Motornyi, Ab initio study of electronic surface states and plasmons of gold: role of the spin-orbit coupling and surface geometry, Ph.D. thesis, Université Paris-Saclay, École Polytechnique, Palaiseau, France (2018).

[32] D. Vanderbilt, "Soft self-consistent pseudopotentials in a generalized eigenvalue formalism," Phys. Rev. B 41, 7892 (1990).

[33] A. Dal Corso, A. Pasquarello, and A. Baldereschi, "Density-functional perturbation theory for lattice dynamics with ultrasoft pseudopotentials," Phys. Rev. B 56, R11369-R11372 (1997).

[34] A. Dal Corso, "Density-functional perturbation the- ory with ultrasoft pseudopotentials," Phys. Rev. B 64, 235118 (2001).

[35] J. Tóbik and A. Dal Corso, "Electric fields with ultrasoft pseudo-potentials: Applications to benzene and anthracene," The Journal of Chemical Physics 120, 99349941 (2004), https://doi.org/10.1063/1.1729853.

[36] B. Walker and R. Gebauer, "Ultrasoft pseudopotentials in time-dependent density-functional theory," J. Chem. Phys. 127, 164106 (2007).

[37] A. Dal Corso and A.M. Conte, "Spin-orbit coupling with ultrasoft pseudopotentials: Application to $\mathrm{Au}$ and $\mathrm{Pt}$," Phys. Rev. B 71, 115106 (2005).

[38] A. Dal Corso, "Density functional perturbation theory for lattice dynamics with fully relativistic ultrasoft pseudopotentials: Application to fcc-Pt and fcc-Au," Phys. Rev. B 76, 054308 (2007).

[39] S. Botti, A. Schindlmayr, R. D. Sole, and L. Reining, "Time-dependent density-functional theory for extended systems," Rep. Prog. Phys. 70, 357 (2007).

[40] X. Qian, J. Li, X. Lin, and S. Yip, "Time-dependent density functional theory with ultrasoft pseudopotentials: Real-time electron propagation across a molecular junction," Phys. Rev. B 73, 035408 (2006).

[41] P. Giannozzi, O. Andreussi, T. Brumme, O. Bunau, M. Buongiorno Nardelli, M. Calandra, R. Car, C. Cavazzoni, D. Ceresoli, M. Cococcioni, N. Colonna, I. Carnimeo, A. Dal Corso, S. de Gironcoli, P. Delugas, R. A. DiStasio Jr.and A. Ferretti, A. Floris, G. Fratesi, G. Fugallo, R. Gebauer, U. Gerstmann, F. Giustino, T. Gorni, J. Jia, M. Kawamura, H.-Y. Ko, A. Kokalj, E. Küçükbenli, M. Lazzeri, M. Marsili, N. Marzari, F. Mauri, N. L. Nguyen, H.-V. Nguyen, A. Otero dela Roza, L. Paulatto, S. Poncé, D. Rocca, R. Sabatini, B. Santra, M. Schlipf, A. P. Seitsonen, A. Smogunov, I. Timrov, T. Thonhauser, P. Umari, N. Vast, X. Wu, and S. Baroni, "Advanced capabilities for materials modelling with QuAntum ESPRESSO," J. Phys.: Condens. Matter 29, 465901 (2017).

[42] A. Dal Corso, The Thermo_pw code is available at https: //dalcorso.github.io/thermo_pw.

[43] We used the PSlibrary (https://dalcorso.github.io/pslibrary). The pseudopotential names were Au.pz-n-rrkjus psl.1.0.0.UPF for the 11-electron US-PP and Au.pz-spn-rrkjus psl.1.0.1.UPF for the 19-electron US-PP.

[44] A. Dal Corso, "Pseudopotentials periodic table: From H to $\mathrm{Pu}$," Computational Materials Science 95, 337 - 350 (2014).

[45] J.P. Perdew, K. Burke, and M. Ernzerhof, "Generalized gradient approximation made simple," Phys. Rev. Lett. 77, 3865 (1996).

[46] J.P. Perdew, K. Burke, and M. Ernzerhof, "Erratum to generalized gradient approximation made simple," Phys. Rev. Lett. 78, 1396 (1997).

[47] A. Alkauskas, S.D. Schneider, C. Hébert, S. Sagmeister, and C. Draxl, "Dynamic structure factors of $\mathrm{Cu}, \mathrm{Ag}$, and $\mathrm{Au}$ : Comparative study from first principles," Phys. Rev. B 88, 195124 (2013).

[48] C. Kittel, Introduction à la physique du solide, 3rd ed. (Dunod, Paris, 1972).

[49] The self-consistent cycle was ended when the estimated energy error was smaller than the threshold of $10^{-12}$ for bulk Au.

[50] G. Prandini, M. Galante, N. Marzari, and P. Umari, 
"SIMPLE code: Optical properties with optimal basis functions," Computer Physics Communications 240, 106 (2019).

[51] M. Schlipf and F. Gygi, "Optimization algorithm for the generation of ONCV pseudopotentials," Computer Physics Communications 196, 36 - 44 (2015).

[52] Pseudopotential was generated using parameters provided in http://bohr.inesc-mn.pt/ jlm/pseudo.html.

[53] To be released in Materials Cloud Archive (2020).

[54] W. Werner, K. Glantschnig, and C. Ambrosch-Draxl, "Optical constants and inelastic electron-scattering data for 17 elemental metals," J. Phys. Chem. Ref. Data 38, 1013 (2009).

[55] W.S.M. Werner, M.R. Went M. Vos, K. Glantschnig, and C. Ambrosch-Draxl, "Measurement and density functional functional calculations of optical constants of $\mathrm{Ag}$ and $\mathrm{Au}$ from infrared to vacuum ultraviolet wavelengths," Phys. Rev. B 77, 161404 (2008).
[56] T. Rangel, T. Kecik, P.E. Trevisanutto, G.-M. Rignanese, H. Van Swygenhoven, and V. Olevano, "Band structure of gold from many-body perturbation theory," Phys. Rev. B 86, 125125 (2012).

[57] We stress that also with the 19-electron NC-PP, the real part of the dielectric function shows two zero-energy crossings, and also in this case the crossing at high energy is more pronounced when crystal local field effects are accounted for: $\operatorname{Re}(\epsilon)$ goes down to about the value of -0.4 while the crossing in the RPA without crystal local field effects, $\operatorname{Re}(\epsilon)$ goes down only to -0.05 .

[58] J.H. Weaver, http://jhweaver.matse.illinois.edu/.

[59] I. Timrov, M. Markov, T. Gorni, M. Raynaud, O. Motornyi, R. Gebauer, S. Baroni, and N. Vast, "Ab initio study of electron energy loss spectra of bulk bismuth up to $100 \mathrm{eV}, "$ Phys. Rev. B 95, 094301 (2017).

[60] I.G. Gurtubay, J.M. Pitarke, I. Campillo, and A. Rubio, "Dynamic structure factor of gold," Computational Materials Science 22, 123 - 128 (2001). 